\title{
Three-dimensional printing-guided percutaneous transcatheter closure of secundum atrial septal defect with rim deficiency: First-in-human series
}

\author{
Zhongmin Wang, Yuhao Liu, Yu Xu, Chuanyu Gao, Yan Chen, Hongxing Luo \\ Department of Cardiology, Zhengzhou University People's Hospital \\ (Henan Provincial People's Hospital), Zhengzhou, Henan, China
}

\begin{abstract}
Background: This study aimed to prospectively evaluate the use of 3-dimensional printing $(3 D P)$ for the percutaneous transcatheter closure of a secundum atrial septal defect (ASD) with rim deficiency less than $5 \mathrm{~mm}$.

Methods: Patients with ASD were scanned using multi-slice computed tomography to acquire raw data for virtual $3 D P$ reconstruction models. Different $A S D$ occluders were tried on the $3 D P$ models to select the optimal size for intraoperative use. The patients were followed up 1 month postoperatively, and 3DP models were again manufactured to observe the operative effects.

Results: From January to April 2016, 6 patients ( 5 females and 1 male) were recruited. Their average age was $29.5 \pm 17.6$ years, and the mean ASD size was $13.4 \pm 1.3 \mathrm{~mm}$. ASD occlusion succeeded in 5 of 6 cases. There were 1,2, and 3 cases with 0-mm distance from the defect rim to the aorta, inferior vena cava, and superior vena cava, respectively. ASD occluder sizes were consistent between preoperative simulation and intraoperative placement in 4 cases. One case had occluder size change from $30 \mathrm{~mm}$ to $34 \mathrm{~mm}$.
\end{abstract}

Conclusions: $A$ 3DP model presents $A S D$ in a more visible way and allows more direct preoperative simulation to choose the most appropriate size of occluder, as compared with conventional imaging techniques. This technique is likely to extend the current indications for ASD with an insufficient rim. (Cardiol J 2016; 23, 6: 599-603)

Key words: three-dimensional printing, percutaneous transcatheter closure, secundum atrial septal defect, preoperative simulation, postoperative evaluation

\section{Introduction}

Atrial septal defect (ASD) affects 0.88-3.89\% of the total population and the incidence rate has increased over time [1]. Secundum ASD is located in the fossa ovalis and surrounding area and accounts for $80 \%$ of ASD [2]. Although surgical closure has been the "gold standard" for ASD, percutaneous transcatheter closure remains the first choice for about $80 \%$ of secundum ASD with a stretched diameter $<38 \mathrm{~mm}$ and sufficient rim of $5 \mathrm{~mm}$ [3]. However, an insufficient rim less than $5 \mathrm{~mm}$ from the inferior vena cava (IVC), superior vena cava (SVC), and aorta remains a contraindication for percutaneous transcatheter closure [4].

Address for correspondence: Dr Hongxing Luo, Department of Cardiology, Zhengzhou University People's Hospital, Zhengzhou, Henan, China, 450003, tel: +86 15290885448, e-mail: 1519782837@qq.com 
Conventional imaging techniques, such as echocardiography, computed tomography (CT), and magnetic resonance imaging provide valuable information for the preoperative evaluation of ASD, but may not be sufficient for optimal preoperative planning of complex ASD cases. Three-dimensional printing (3DP) is a novel technology that fabricates a tactile object with raw materials through layer-by-layer deposition. 3DP has been used for preoperative evaluation and device development in orthopedics, plastic surgery, general surgery, and other areas [5-7]. However, no studies have reported to date using of 3DP for preoperative evaluation of ASD with defect rim less than $5 \mathrm{~mm}$.

It was hypothesized that a 3DP heart model would aid in the percutaneous transcatheter closure of secundum ASD by allowing more precise demonstration of anatomical structures and more patient-specific preoperative simulation, as compared with conventional 2-dimensional medical images. This article presents a preliminary study on 6 patients who had secundum ASD defect rim less than $5 \mathrm{~mm}$ from the IVC, SVC, or aorta.

\section{Methods}

This study was approved by the Institutional Review Board of Henan Provincial People's Hospital and is in compliance with the human research regulations stated in the Declaration of Helsinki.

\section{Inclusion and exclusion criteria}

A patient was included if the following criteria were satisfied: 1) they were capable of providing written informed consent; 2) color flow Doppler showed a distance from ASD rim to aorta, SVC, or IVC less than $5 \mathrm{~mm}$, with a left-to-right shunt; and 3) physical examination revealed systolic murmurs located between the second and third ribs to the left of the sternum. Exclusion criteria were severe kidney dysfunction or arrhythmias, such as atrial fibrillation or frequent premature contractions that were difficult to control at fewer than $70 \mathrm{bpm}$ using medications, such as beta-blockers.

\section{Computed tomography acquisition and three-dimensional virtual reconstruction}

Before 64-slice CT (Siemens AG, Berlin, Germany), the patient was required to have a heart rate less than $70 \mathrm{bpm}$; otherwise, $25-50 \mathrm{mg}$ metoprolol was given $1 \mathrm{~h}$ before scanning. Patients were placed in supine position with both hands beneath the head. Sagittal and coronal scans of the whole heart were first performed with the patient in inspiratory breath-hold. Then iopamidol $100 \mathrm{~mL}$ was intravenously injected at $5.0 \mathrm{~mL} / \mathrm{s}$ through the median cubital vein with a saline chase of $15 \mathrm{~mL}$ at $5.0 \mathrm{~mL} / \mathrm{s}$. Images were acquired with a gantry rotation time of $0.35 \mathrm{~s}$ and tube current $200 \mathrm{~mA}$ at $120 \mathrm{kV}$, with dose modulation of $75 \%$ of RR interval. All images were reconstructed with slice thickness of $0.625 \mathrm{~mm}$ at the workstation, and then saved in digital imaging and communications in medicine (DICOM) format.

The DICOM file was read into the Mimics 17.0 software (Materialise NV, Leuven, Belgium). A mask was generated with the threshold of 863-3,010 Hounsfield Units. Then the mask was regionally grown and edited to remove the noncardiac neighboring parts. The remaining cardiac mask was calculated for a 3D model that was later smoothed and saved in standard tessellation language (.stl) format.

\section{Three-dimensional printing}

The .stl file was loaded into a ZRapid SLA450 RAPID 3D printer (ZRapid Company, Jiangsu, China) and printed out with ZR80 photoreactive resin polylactic acid (ZRapid Company, Jiangsu, China) using stereolithography technique. Briefly, a helium-cadmium laser generator controlled by a computer emitted an ultraviolet beam to scan the fluid surface of a flume containing fluid photosensitive resin in a dot-by-dot manner. As a result, the scanned region underwent a photopolymerization reaction and solidified in a few seconds to a thin layer. Then the work elevator moved down by $1 \mathrm{~mm}$ so that the solidified layer was again immersed in liquid resin. A milling cutter removed the redundant solidified resin and a new layer was solidified again following the above-mentioned steps, until the whole heart model was completely manufactured. The resolution of the printer was $0.1 \mathrm{~mm}$. Time of printing was mainly dependent on object size.

\section{Preoperative simulation}

We tried different sizes of ASD occluders on the 3DP heart model. An occluder was defined as "appropriate" if 1) had no compression on adjacent tissues including the tricuspid valve, and 2) did not obstruct the blood flow from SVC or IVC.

\section{Operative protocols}

Patients were placed in supine position and locally anesthetized with $1 \%$ lidocaine. After intravenous injection of $100 \mathrm{U} / \mathrm{kg}$ heparin, a Beijing ASD occluder (Starway Medical Technology, Inc., Bei- 
Table 1. Summary of patient characteristics and occluder size.

\begin{tabular}{|c|c|c|c|c|c|c|c|c|}
\hline \multirow[t]{2}{*}{ No. } & \multirow[t]{2}{*}{ Age } & \multirow[t]{2}{*}{ Sex } & \multirow{2}{*}{$\begin{array}{c}\text { ASD size } \\
{[\mathrm{mm}]}\end{array}$} & \multicolumn{3}{|c|}{ Distance [mm] } & \multicolumn{2}{|c|}{ Occluder size [mm] } \\
\hline & & & & SVC & IVC & AO & $\begin{array}{l}\text { Preoperative } \\
\text { simulation }\end{array}$ & $\begin{array}{c}\text { Final } \\
\text { placement }\end{array}$ \\
\hline 1 & 11 & Female & 15.0 & 0 & 15.6 & 0 & 26 & 26 \\
\hline 2 & 27 & Female & 14.5 & 33.5 & 0 & 16.0 & 30 & 34 \\
\hline 3 & 34 & Female & 14.0 & 18.4 & 0 & 13.0 & 28 & $N / A^{*}$ \\
\hline 4 & 47 & Female & 11.8 & 2.6 & 16.6 & 0 & 20 & 20 \\
\hline 5 & 50 & Female & 13.0 & 8.3 & 19.0 & 0 & 24 & 24 \\
\hline 6 & 8 & Male & 12.0 & 6.3 & 20.0 & 0 & 20 & 20 \\
\hline
\end{tabular}

*Cardiac catheterization showed pulmonary vascular resistance higher than 20 Wood Units, and ASD closure was abandoned; AO — aorta; ASD — atrial septal defect; IVC — inferior vena cava; SVC — superior vena cava

jing, China) was placed through a standard femoral venous sheath. The occluder was pushed and pulled several times to test its stability. The ASD occluder was then examined by chest radiography and bedside echocardiography to observe the morphology and position of the occluder. After the intervention, the puncture site was compressed with a sand bag for $4 \mathrm{~h}$ and the patient was required to remain at bed rest for $12 \mathrm{~h}$. Postoperatively, the patient was intravenously injected with $400-500 \mathrm{IU} / \mathrm{kg}$ low-molecular-weight heparin every $12 \mathrm{~h}$ in two doses. Oral aspirin or clopidogrel were given for the first 6 months to prevent thrombosis.

\section{Postoperative follow-up}

One month postoperatively, the patients were re-examined by echocardiography and multi-slice computed tomography (MSCT). As mentioned above, the MSCT dataset was 3-dimensionally reconstructed, and then a 3DP heart model was produced for postoperative evaluation.

\section{Statistical analysis}

All data were processed using SPSS 19.0 software (IBM Corporation, New York, US). Continuous data were expressed as mean \pm standard deviation.

\section{Results}

From January to April 2016, 6 patients were recruited for our study. Eight heart models were generated using 3DP technology, including 2 postoperative heart models.

Table 1 summarizes patient demographics, echocardiographic parameters, and occluder sizes during preoperative simulation and intraoperative placement. There were 5 females and 1 male. Their average age was $29.5 \pm 17.6$ years, ranging from 8 to 50 years. The mean ASD size was $13.4 \pm 1.3 \mathrm{~mm}$. One patient had mixed ASD with $0 \mathrm{~mm}$ from the defect rim to the aorta and SVC. Two patients had $0-\mathrm{mm}$ distance from the defect rim to the IVC. Three patients had 0 -mm distance from the defect rim to the aorta. The ASD occluder sizes were consistent in 4 of 6 cases. Case 2 was placed with a $34-\mathrm{mm}$ ASD occluder, which was larger than the $30-\mathrm{mm}$ occluder predicted by preoperative simulation. Case 3 had pulmonary vascular resistance higher than 20 Wood Units during right heart catheterization and thus the interventional closure was discontinued. Pulmonary vasodilator therapy including sildenafil and bosentan was given to lower the pulmonary pressure.

Figure 1 shows the workflow to fabricate a 3DP heart model for preoperative simulation in the representative Case 1.

\section{Discussion}

The last decade has seen the development of 3 DP technology in cardiology. In 2006, Noecker et al. [8] first printed pediatric heart models using CT datasets and suggested that the 3D models were likely to aid in the placement of medical devices. Two years later, Kim et al. [9] employed 3DP models in the preoperative evaluation of ventricular septal defect, atrial septal defect, artificial mitral valve perivalvular leakage, and thoracic aortic pseudoaneurysm. In 2015, they reviewed their single-center experience using 3DP for preoperative evaluation and concluded that $3 \mathrm{DP}$ could reduce the mortality and morbidity of complicated cardiac surgeries [10]. None of these preliminary 


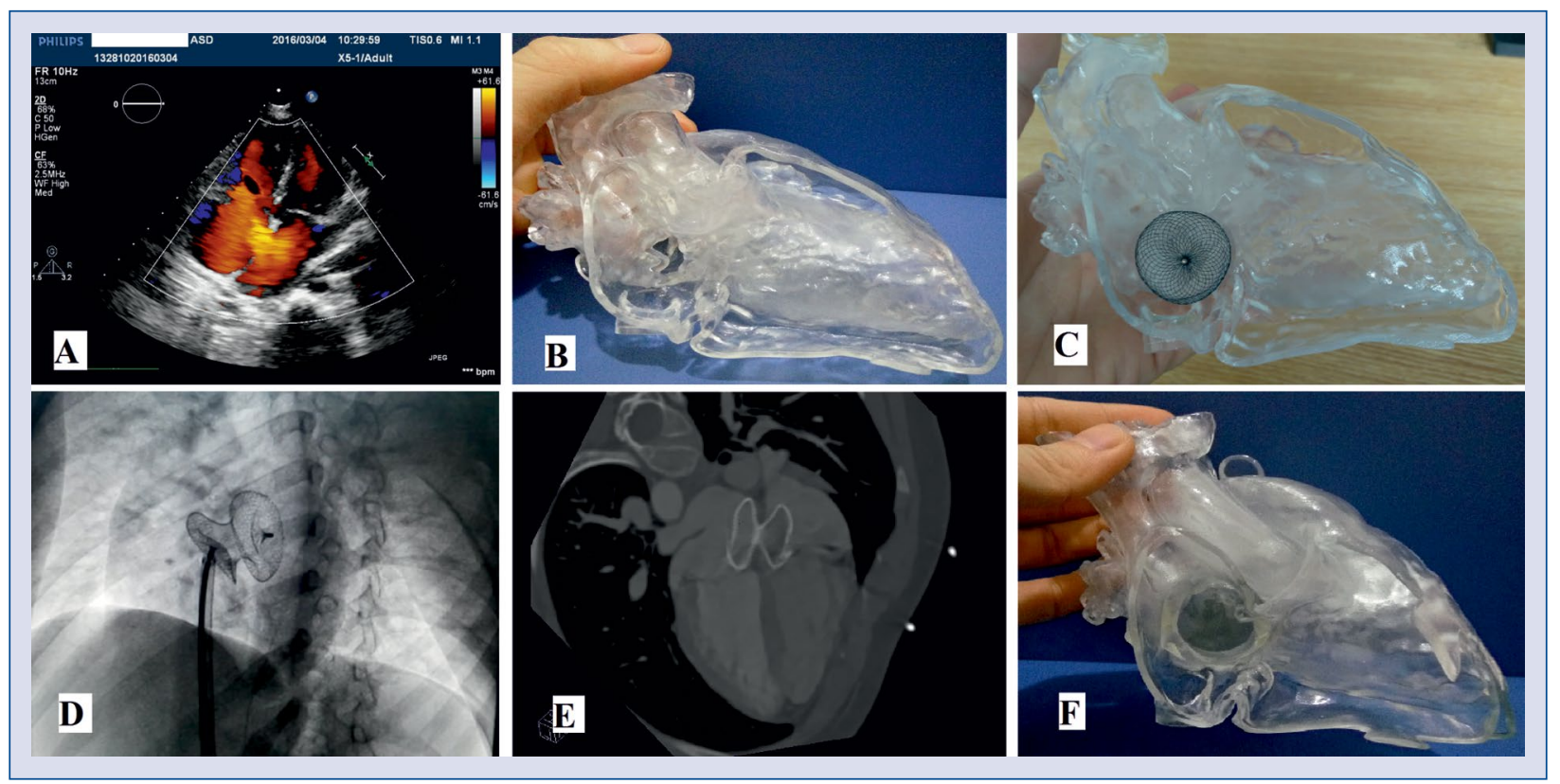

Figure 1. Workflow from echocardiographic evaluation to postoperative three-dimensional printing (3DP) assessment; A. Preoperative color flow Doppler shows left-to-right shunt across the defect during systole; B. A 3DP heart model shows a mixed-type atrial septal defect (ASD) with $0-\mathrm{mm}$ distance from defect rim to aorta and superior vena cava (SVC). Most cardiac anatomical structures, e.g., atrium, ventricle and interventricular groove, are shown in the 3DP heart models, but it is noteworthy that no heart valves are demonstrated in the model; C. During preoperative simulation, a 26-mm ASD occluder was placed to completely cover the defect area without obvious obstruction of blood flow from major blood vessels including SVC, inferior vena cava (IVC), and aorta; D. A 26-mm ASD occluder was placed after only first attempt. An intraoperative push-and-pull test demonstrated good stability of the occluder; E. Postoperative multi-slice computed tomography showed no device misalignment or impingement on adjacent structures, e.g., aortic valves, SVC, or IVC; F. The postoperative 3DP heart model visually demonstrated the relationship between the ASD occluder and adjacent structures. The ASD occluder completely covered the entire defect without impingement of adjacent tissues.

studies, however, have focused on the application of 3DP to ASD with insufficient defect rim near major blood vessels, including IVC, SVC, and aorta.

Results showed that it is feasible to employ 3DP to optimize the choice of occluders for ASD with an insufficient defect rim. ASD occluders were successfully placed in 5 of 6 cases. The occluder sizes were consistent between preoperative simulation and intraoperative placement in 4 of 6 cases. However, 2 cases with 0 -mm distance from defect rim to IVC were not easily treated. Case 3 with pulmonary artery hypertension had a contraindication to closure. In case 2 , the $30-\mathrm{mm}$ ASD occluder was replaced by a $34-\mathrm{mm}$ device. This was possibly the result of the weight of the ASD occluder, which slipped from the original site more easily when the IVC had no rim. Although Yan et al. [11] also demonstrated the feasibility of a 3DP heart model in a secundum ASD case with 0 -mm distance from defect rim to SVC and IVC.The results of this study indicated that the procedure may potentially have high risk, even with a 3DP heart model, since Case 2 was finally treated with a larger ASD occluder and Case 3 had an occluder contraindication.

Overall, the 3DP heart model presents a more visible and comprehensive 360-degree view for better understanding of cardiac anatomical structures (e.g., SVC, IVC, and aorta), allowing more effective and individualized preoperative planning by trying different sizes of ASD occluders preoperatively. 3DP technology is likely to reduce intraoperative time and postoperative complications, and increase success rates through a more efficient preoperative simulation. Some open heart surgeries for the repair of ASD may be avoided after a thorough preoperative evaluation to choose an appropriate occluder. It is currently difficult to define the exact indications for printing a heart model for preoperative planning. However, based on our experience, we propose the adoption of this technology for non-routine procedures after fully 
discussing the details with the patient and obtaining informed consent.

The current application of 3D printing in medicine is still limited by the following factors. First, the images acquired by $\mathrm{CT}$ or magnetic resonance imaging are not precise enough for 3DP, which has a minimum resolution of $0.01 \mathrm{~mm}$. This problem may be resolved with the future development of medical imaging techniques. It was also noted that the 3DP heart model shows no heart valves, which might be due to its thinness and dynamic movements. Color Doppler is capable of showing both heart valves and blood flow, and thus may be integrated to improve the quality of images [12]. Second, the cost-effectiveness analysis should be taken into consideration when a cardiologist plans to use a 3DP heart model for preoperative simulation and postoperative evaluation. Patients have to pay extra not only for 3DP per se (200 to 500 USD, depending on the materials and weight of the model), but also for preoperative and postoperative MSCT scanning, as well as prolonged hospitalized stays. Situations still exist in which an experienced interventional cardiologist is capable of performing a successful operation in a complex case without the help of a 3DP heart model. Thus, the decision to print a heart model should be discussed with an experienced cardiologist and based on the patient's ability to pay. 3DP may be more important for less experienced hospitals and for more challenging cases, such as fenestrated ASD with large atrial septal aneurysm. Third, since the research on the applications of 3DP technology in clinical medicine is in its infancy, most studies have been single case reports, which are insufficient to assess the actual effects of 3DP on prognosis and complications. Thus far, we have not observed any adverse events in the 5 cases implanted with an ASD occluder. A large-scale clinical trial with long-term followup may reveal the positive or negative effects of 3DP technology on patient survival.

\section{Conclusions}

In conclusion, the preliminary trials have demonstrated for the first time that 3DP is feasible for preoperative planning of pediatric and adult ASD with insufficient defect rim less than $5 \mathrm{~mm}$ from the SVC, IVC, or aorta. However, a randomized controlled trial with more patients is necessary to test whether or not 3DP technology helps to extend the indications for ASD in current guidelines.
Sources of funding: This work has been supported by the Henan Provincial Key Medical Program (Henan Provincial Department of Science and Technology) and the Henan Provincial New Medical Technology Program (Health and Family Planning Commission of Henan Province).

\section{Conflict of interest: None declared}

\section{References}

1. Marelli AJ, Mackie AS, Ionescu-Ittu R, Rahme E, Pilote L. Congenital heart disease in the general population: Changing prevalence and age distribution. Circulation, 2006; 115: 163-72. doi: 10.1161/CIRCULATIONAHA.106.627224.

2. Baumgartner H, Bonhoeffer P, De Groot NMS et al. ESC Guidelines for the management of grown-up congenital heart disease (new version 2010): The Task Force on the Management of Grown-up Congenital Heart Disease of the European Society of Cardiology (ESC). Eur Heart J, 2010; 31: 2915-2957. doi: 10.1093/eurheartj/ehq249.

3. Warnes CA, Williams RG, Bashore TM et al. ACC/AHA 2008 Guidelines for the management of adults with congenital heart disease: A report of the American College of Cardiology/American Heart Association Task Force on Practice Guidelines (Writing Committee to Develop Guidelines on the Management of Adults With Congenital Heart Disease): Developed in Collaboration With the American Society of Echocardiography, Heart Rhythm Society, International Society for Adult Congenital Heart Disease, Society for Cardiovascular Angiography and Interventions, and Society of Thoracic Surgeons. Circulation, 2008; 118: e714-e833. doi: 10.1016/j.jacc.2008.10.001.

4. Fischer G, Stieh J, Uebing A, Hoffmann U, Morf G, Kramer HH. Experience with transcatheter closure of secundum atrial septal defects using the Amplatzer septal occluder: A single centre study in 236 consecutive patients. Heart, 2003; 89: 199-204. doi: PMC1767528.

5. Yu AW, Duncan JM, Daurka JS, Lewis A, Cobb J. A feasibility study into the use of three-dimensional printer modelling in acetabular fracture surgery. Advances in Orthopedics, 2015; 2015: 1-4. doi: 10.1155/2015/617046.

6. Kamali P, Dean D, Skoracki R et al. The current role of threedimensional (3D) printing in plastic surgery. Plast Reconstr Surg, 2016: 1. doi: 10.1097/PRS.0000000000000150.

7. Igami T, Nakamura Y, Hirose T et al. Application of a threedimensional print of a liver in hepatectomy for small tumors invisible by intraoperative ultrasonography: Preliminary experience. World J Surg, 2014; 38: 3163-3166. doi: 10.1007/s00268014-2740-7.

8. Noecker AM, Chen J, Zhou Q et al. Development of patientspecific three-dimensional pediatric cardiac models. ASAIO J, 2006; 52: 349-353. doi: 10.1097/01.mat.0000217962.98619.ab.

9. Kim MS, Hansgen AR, Wink O, Quaife RA, Carroll JD. Rapid prototyping: A new tool in understanding and treating structural heart disease. Circulation, 2008; 117: 2388-2394. doi: 10.1161/ CIRCULATIONAHA.107.740977.

10. Schmauss D, Haeberle S, Hagl C, Sodian R. Three-dimensional printing in cardiac surgery and interventional cardiology: A single-centre experience. Eur J Cardio-Thorac, 2015; 47: 1044-1052. doi: 10.1093/ejcts/ezu310.

11. Yan C, Li H, Sun X. Three-dimensional printing as an aid in transcatheter closure of secundum atrial septal defect with rim deficiency. Circulation, 2016; 133: e608-e610. doi: 10.1161/CIRCULATIONAHA.115.020735.

12. Samuel BP, Pinto C, Pietila T, Vettukattil JJ. Ultrasound-derived three-dimensional printing in congenital heart disease. J Digit Imaging, 2015; 28: 459-461. doi: 10.1007/s10278-014-9761-5. 